\title{
Analogy to mechanics for introducing optical imaging and teaching about astigmatism
}

Pietro Ferraro

Pietro Ferraro, "Analogy to mechanics for introducing optical imaging and teaching about astigmatism," Proc. SPIE 3190, Fifth International Topical Meeting on Education and Training in Optics, (8 December 1997); doi: $10.1117 / 12.294416$

Event: Fifth International Topical Meeting on Education and Training in Optics, 1997, Delft, Netherlands 
Analogy with mechanics to introduce optical imaging and to teach astigmatism

Pietro Ferraro

\author{
Istituto di Cibernetica del CNR, Via Toiano n.6, 80072,Arco Felice (Na), Italy. \\ and \\ Istituto Professionale di Stato per l'Industria e l'Artigianato "G.L.Bernini" \\ Via Arco Mirelli 19/a Napoli, 80122 Italy
}

\begin{abstract}
A simple analogy between mechanics and optics has been found for introducing imaging proprieties of lenses. In this analogy the light beam in optics has the role of a sample beam in mechanics; while in optics the light beam is expanded or reduced in size by the power of the lens in mechanics the sample beam suffer elongation when a tensile or compressive stress is applied. Following the analogy the aberration introduced by the lens can be the corespondent non linear effect that occurs in mechanics when elastic limit is over. Using the simple developed analogy astigmatism aberration of cylindrical lenses can be explained trough a very simple classroom demonstration using rubber stripes.
\end{abstract}

Keywords: lenses, astigmatism, geometrical optics. mechanics analogy.

\title{
1. INTRODUCTION
}

In teaching physics it is useful, whenever possible, to use the analogies that can be found between models of interpretation of different physical situations. In this paper I establish relationships between two basic and simple phenomena that I never had found in the pedagogical literature. Optics and mechanics are considered two separate areas of the physics, but, by observing them in more depth, some situations and interesting relationships can be found and analyzed. This is the case for a light beam and a bar of homogeneous material. Although the two have no apparent connection to each other the effect of magnification produced on the light beam by a telescopic optical system could be seen as analogue to the behaviour of the material when it is subjected to tensile or compressive force.

\section{MECHANICS: HOOKE'S LAW}

When a rod of length $l$ and cross-section $A$ is stretched by a force $F$ it will suffer an elongation $e=l^{\prime}-l$ where $l^{\prime}$ is the length of the rod as produced by the applied force $F ; e$ is proportional to the applied force $F$ according to the theory of the elasticity based on the Hooke's law expressed by the following equation

$$
\frac{F}{A}=\mathrm{Y} \frac{e}{l}
$$

where $\mathrm{Y}$ is the proportionality constant called Young's modulus ${ }^{1}$. Sometimes the eq.(1) is found in a different form

$$
\sigma=\mathrm{Y} \varepsilon
$$

where $\sigma=F / A$ is the stress, the force for unit area; $\left.\varepsilon=l^{\prime}-l\right) / l$ is the strain, the elongation per unit length.

\section{OPTICS: BEAM EXPANSION BY A TELESCOPE}

Referring to drawing of Fig.1, we can consider, an incoming collimated light beam of diameter $D$. When the beam is imaged by a Galielean telescopic system made by a diverging lens with focal length $f_{1}$ and a convergent lens with focal length $f_{2}$, it is magnified. It is well known that for Galilean telescope the magnification is given by

$$
G=\frac{f_{2}}{f_{1}}
$$

The magnification $G$ is the ratio between the magnified diameter $D^{\prime}$ and the unmagnified diameter $D$ of the incoming light beam. It is easy to show by simple calculation that eq.(3) can be transformed in the following form 


$$
\frac{D^{\prime}-D}{D}=\frac{\Delta}{f_{1}}
$$

where $\Delta=f_{2}-f_{1}$ is the distance between the two lenses.

\section{ANALOGY}

The left-hand side of eq.(3) we can describe as the imaging strain experienced by the light beam; infact the quantity $\left(D^{\prime}\right.$ $D) / D$ assumes the role analogous to the mechanical strain $\varepsilon$ to which a specimen is subjected when a force is applied to stretch it. In this way we can make a comparison between the eq.(2) and eq.(4) for comparing mechanical strain and imaging strain

$$
\begin{aligned}
& \varepsilon_{\text {mech }}=\frac{l^{\prime}-l}{l}=\frac{\sigma}{\mathrm{Y}} \\
& \varepsilon_{\text {opt }}=\frac{D^{\prime}-D}{D}=\frac{\Delta}{f_{1}}
\end{aligned}
$$

We may recognize the correspondence between the mechanical stress $\sigma$ and the ratio $\Delta / f_{1}$, if we can assume that the analogue of the Young's modulus in optics is a unit constant. We are not considering the intrinsic characteristics of the light beam as wavelength, polarisation, irradiance.

The correspondence between $\Delta / f_{1}$ and $\sigma$ is explained as follows: if more elongation is wanted larger stress $\sigma$ has to be applied to the specimen increasing the force $F$. If a larger diameter light beam is required, it can be obtained increasing the distance $\Delta$ or reducing the focal length $f_{1}$. In this way we can try to define $\Delta / f_{1}$ as imaging stress. The analogy is perfectly well sustained also when the stress $\sigma$ changes its sign. Infact depending form the sign of $\sigma$ it is known that the specimen is subject to a tensile or a compressive stress, producing elongation or compression along the direction of the applied stress (Fig.2). In optics the expansion or compression of the light beam is obtained when the telescope is inverted in respect to the direction of propagation of the beam. In this case the sign of $\Delta=f_{2}-f_{1}$ changes.

\section{TEACHING ASTIGMATISM}

The Ronchi Test is a experimental method to study aberrations of optical systems. When this test is applied in presence of pure astigmatism, an interesting effect called "capriola" takes place ${ }^{2}$. "Capriola" is an Italian word that means somersault or caper in English. Using the proposed simple analogy it is possible to facilitate understanding of the "capriole" effect and to teach optics of cylindrical lenses and astigmatism. A simple demonstration, by applying a force to a rubber band, is proposed to simulate the "capriola" effect.

Introducing to my students cylindrical optics and their imaging proprieties I noted they were not completely satisfied. Some students did not understand what happens along the two different directions in the image plane. They had difficulty to visualize the three-dimensional representation because the asymmetry of such optical systems. Furthermore they had difficulties to understand that magnification can be different along two axis and the implications of such asymmetry on produced images. The asymmetry of cylindrical lenses is an intrinsically difficulty encountered in teaching astigmatism and additional tools or demonstrations have been developed ${ }^{3}$ to facilitate understanding of the subject.

\subsection{Observing "Capriole"}

A simple demonstration can be performed to show "capriole" using a telescope, made of two cylindrical lenses (Fig.2), and a transmission grating made of parallel lines. When the grating is projected by collimated light beam and imaged trough a telescopic optical system an image of grating can be observed on a CCD array or on a screen. If lines of the grating, are inclined with respect to the axis of the cylindrical lenses the projected image of the grating will appear rotated. Furthermore if the magnification of the telescope is varied the lines of the imaged grating will appear, inclined at different angles, and "capriole" can be observed!

In introductory optics courses, students learn that optical elements such lenses and mirror, are used generally to obtain, for example, magnified image of an object but certainly not to obtain rotated image of it, as at first glance it could appear in the "capriole". When a student try to explain how the "capriola" occurs, often he forget that an optical system gives a 
magnified (enlarged or reduced size) image having same proportions of the object only in the special case of circular symmetry of the optical system around its optical axis. A cylindrical lens has no such a symmetry and so the resulting image will be magnified only along one direction: the $y$-axis that is perpendicular to the axis of the cylinder. To explain "capriole", it is useful consider how a cylindrical lens can image a grating in three different cases: lines that are respectively parallel, orthogonal and making an angle with the an axis that is parallel to the axis of the cylindrical lenses.

- Lines of the grating are parallel to the x-axis: depending from the distance between the grating and the lens, an observer will see a magnified image of the grating lines (thicker or thinner lines) at different image planes, because the lens has a magnifying effect only in a direction perpendicular to the lines (Fig.3a).

- Lines of the grating are parallel to the y-axis: since the lens has a focal power in planes that contain the direction of lines, the image will be magnified only along that directions; imaged lines will be shorter or longer but unchanged in thickness and spacing. If lines are long compared to the aperture of the lens and are continuos and regular, the image will appear identical to the object and the lens seems to have no effect! (Fig.3b).

- Lines of the grating make an angle $\theta$ with axis of the cylinder, with $0^{\circ}<\theta<90^{\circ}$ : the resulting image will appear rotated. In the fig. $3 \mathrm{c}$ is shown a "capriola". To explain the phenomena we will refer to the fig. 2 . When a cylindrical lens is used, the size of the object along the $y$-axis is optically magnified while the size of the object along the $x$-axis does not; it will be formed an image with thinner or thicker lines, and respectively reduced or increased spacing, but inclined at different angle. Since the amount of magnification along the y-axis changes with the distance lens-object, when the lens is translated along its optical axis, while the grating is fixed, an observer will see what seems to be a rotation of the lines, giving rise to the "capriole". Rotation observed in the "capriola" effect can also be explained by using vectors: given a vector in a plane, if one component is reduced or increased, while the other remains constant, rotation will result.

\section{2 "Capriole" in mechanics by using a rubber band}

The proposed analogy between the mechanics and optics can be useful to explain the apparent rotation of lines imaged by a cylindrical lens. With this approach students can touch with their own hands how the effect of magnification along one axis can cause the "capriola" effect. To perform such demonstration is a rubber band on which a grating made of parallel lines has been previously drawn have to be used. It could be opportune to prepare three rectangular rubber bands. On each rubber band lines should be drawn at a different angle, respectively $0^{\circ}, 90^{\circ}$ and $45^{\circ}$. Applying a tensile force along the longer side of the rubber band it is possible to simulate "capriole" observed by the cylindrical lens. The rubber band will experience an elongation only along the direction of the applied force; one more time three different situations can be distinguished:

- when the direction of the applied force is orthogonal to the lines, their thickness and spacing will change without affecting orientation angle (Fig.4);

- when the direction of the tensile force is parallel to the lines, the only effect will be the elongation of the lines, but neither lines inclination nor their thickness will change;

- when the direction of the applied force makes an angle $\theta$ with the lines, then a similar "capriola" effect observed in optics takes place. In this case lines will be elongated only along the direction of the applied force while the other direction will not be affected; in this case the explanation given for the "capriole" in optics is still valid for the rubber band (Fig.5) and vice versa.

The advantage of using this analogy lies in the fact that students can use their intuitive knowledge in mechanics to understand optics of cylindrical lenses. Infact they easily foresee what happens to a rubber stripe when a tensile stress is applied to it, but, as I have experienced, they are not so able about the "capriole".

Furthermore, limits of validity of the physics model are for mechanics the elastic limit and the paraxial limit for the optics case. Beyond the said limits the linear eqs(2) and (4) are no longer valid and in both cases non-linear effect must be taken into account: plastic deformation effect in the specimen and aberrations for the light beam.

\section{ACKNOWLEDGEMENTS}

The author is grateful to Andrea Finizio for providing figures 3,4,5.

\section{REFERENCES}

1. E..J. Hearn, "Mechanics of Materials" Pergamon Press, 1977; pp.2-4.

2. Extensive references on the subject of Ronchi test can be found in: D.Malacara, Editor, Optical

shop testing, 2nd edition, John Wiley \& Sons, New York (1992) 321-365.

3. M.Riegel and B.Holmes "Model of astigmatism", Phys. Teach., 26, 96 (Dec.1988). 

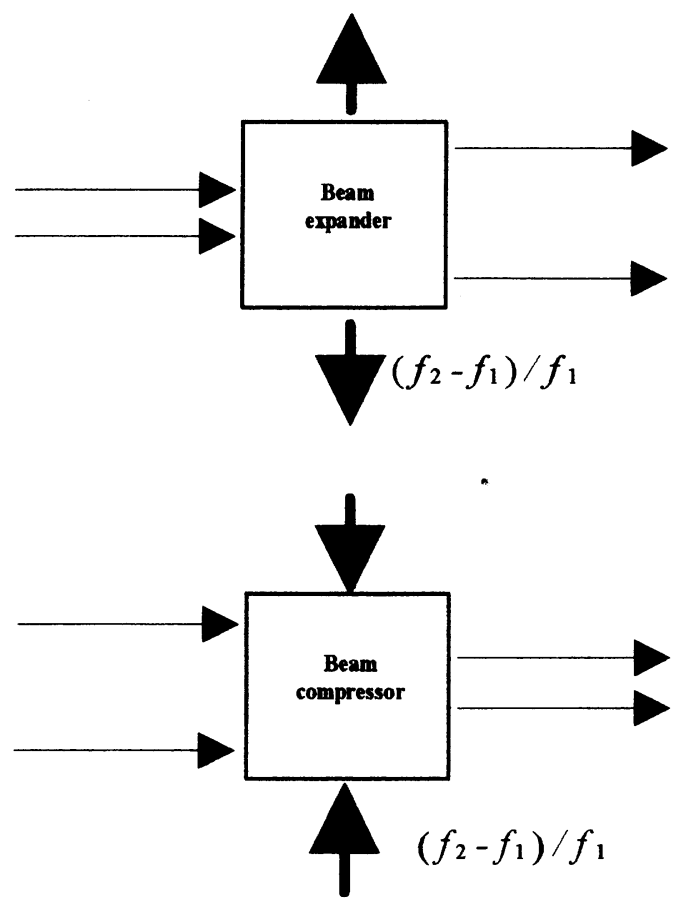

Fig. 1 Beam expansion by a telescope; reversing the telescope the beam is compressed.

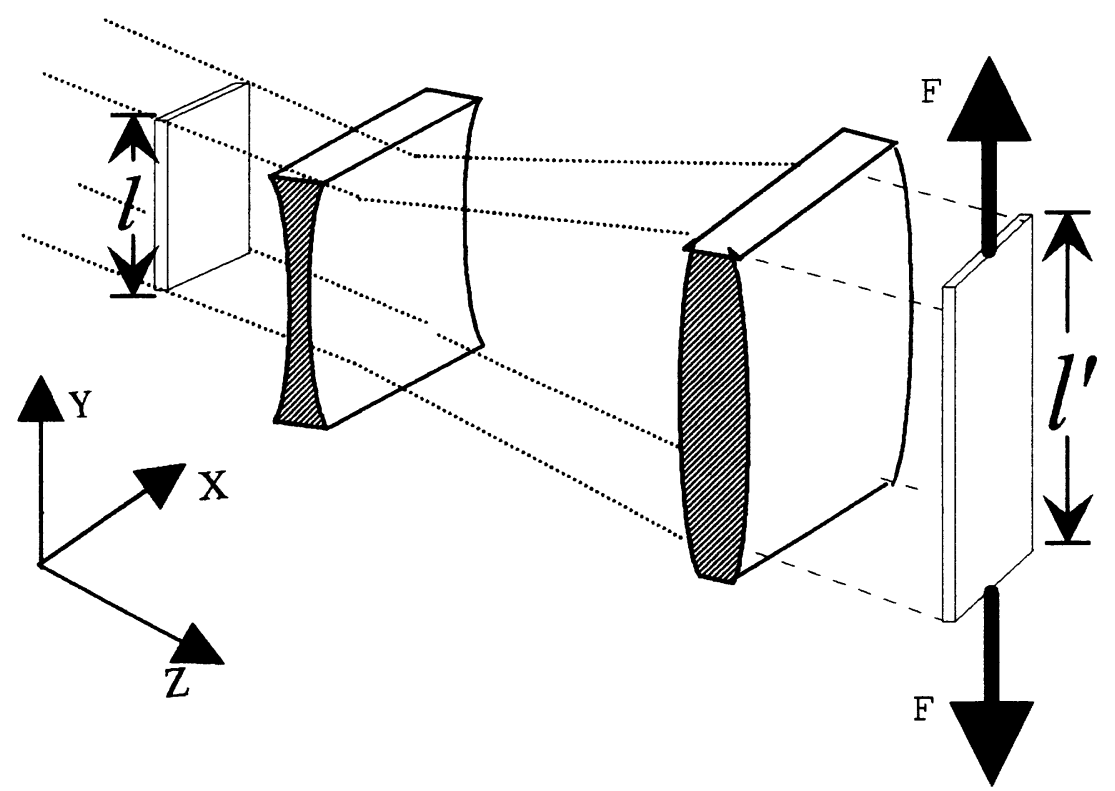

Fig.2 Qualitative illustration of the analogy between a a stripe subjected to tensile force and the imaging propriety of a cylindrical telescope system on a collimated light beam. It is possible to define a the imaging strain and image stress in analogy to mechanics. 


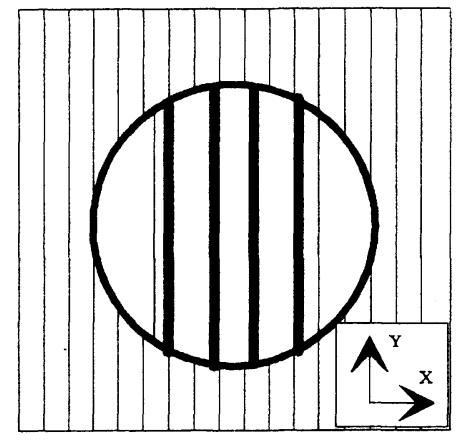

(a)

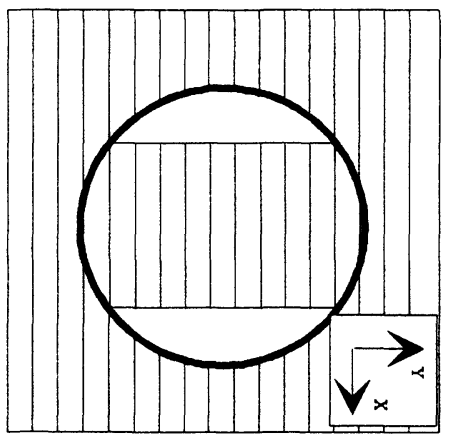

(b)

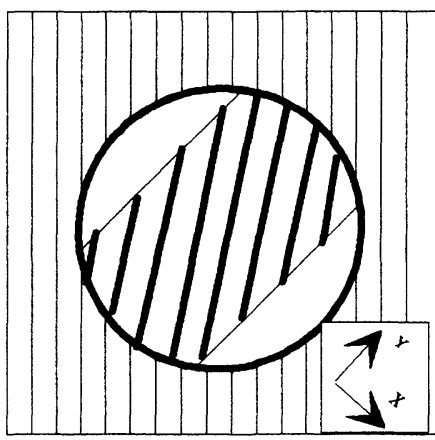

(c)

Fig.3 Imaging a grating by a cylindrical telescope: (a) when lines are parallel to the axis of cylinder, the image is magnified only along direction that is orthogonal to the direction of lines; (b) if lines are orthogonal to the axis of cylinder, lines are magnified along their own direction and the image appears identical to the object! (c) lines are oriented at $45^{\circ}$, image is magnified only along $x$-axis and appears rotated; if the power of the telescope is changed rotation will appear producing "capriole".

(a)

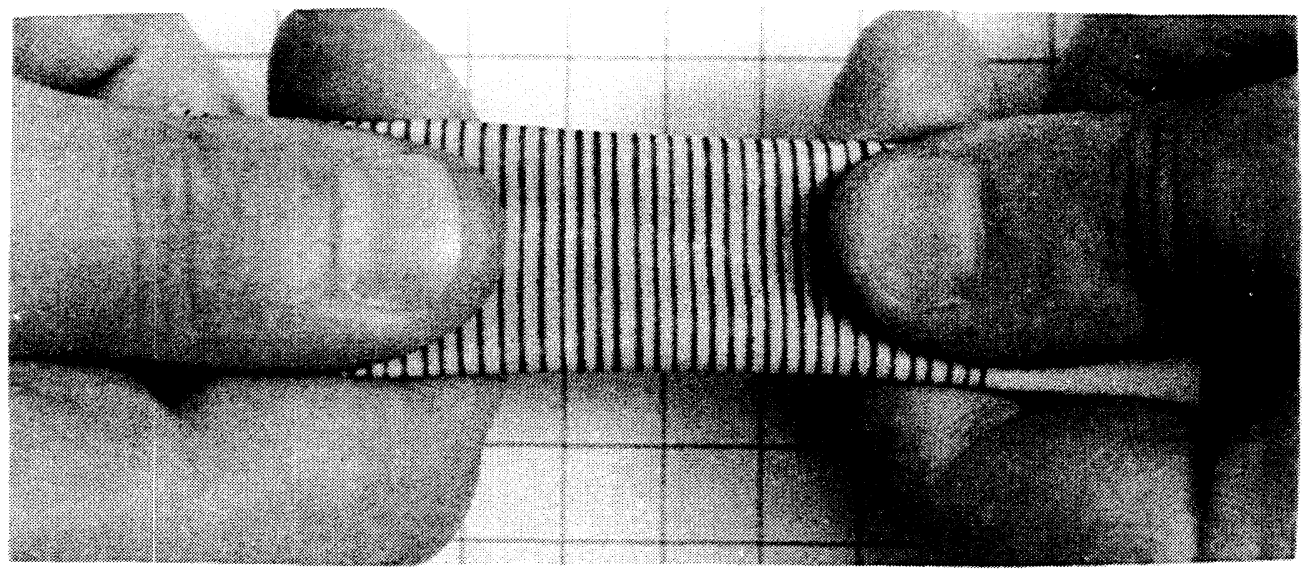

(b)

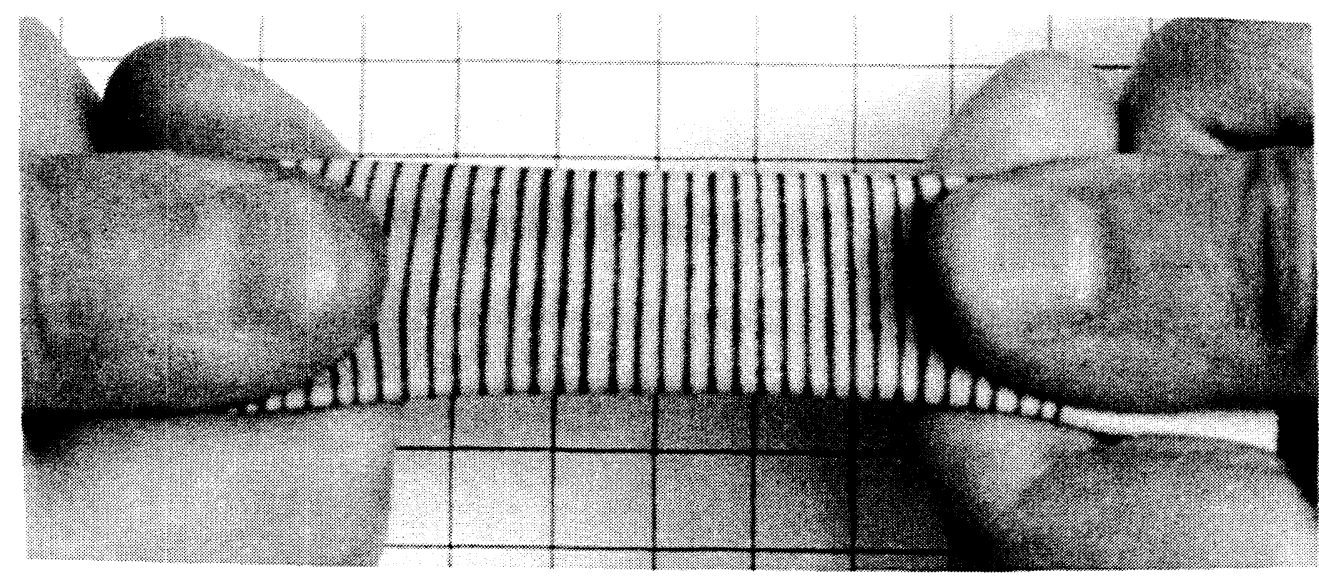

Fig.4 A rubber band with lines parallel to the short side. (a) no force applied; (b) when a tensile force is applied along the longer side: lines appear with increased thickness and spacing simulating the situation shown in figure $3 a$. 

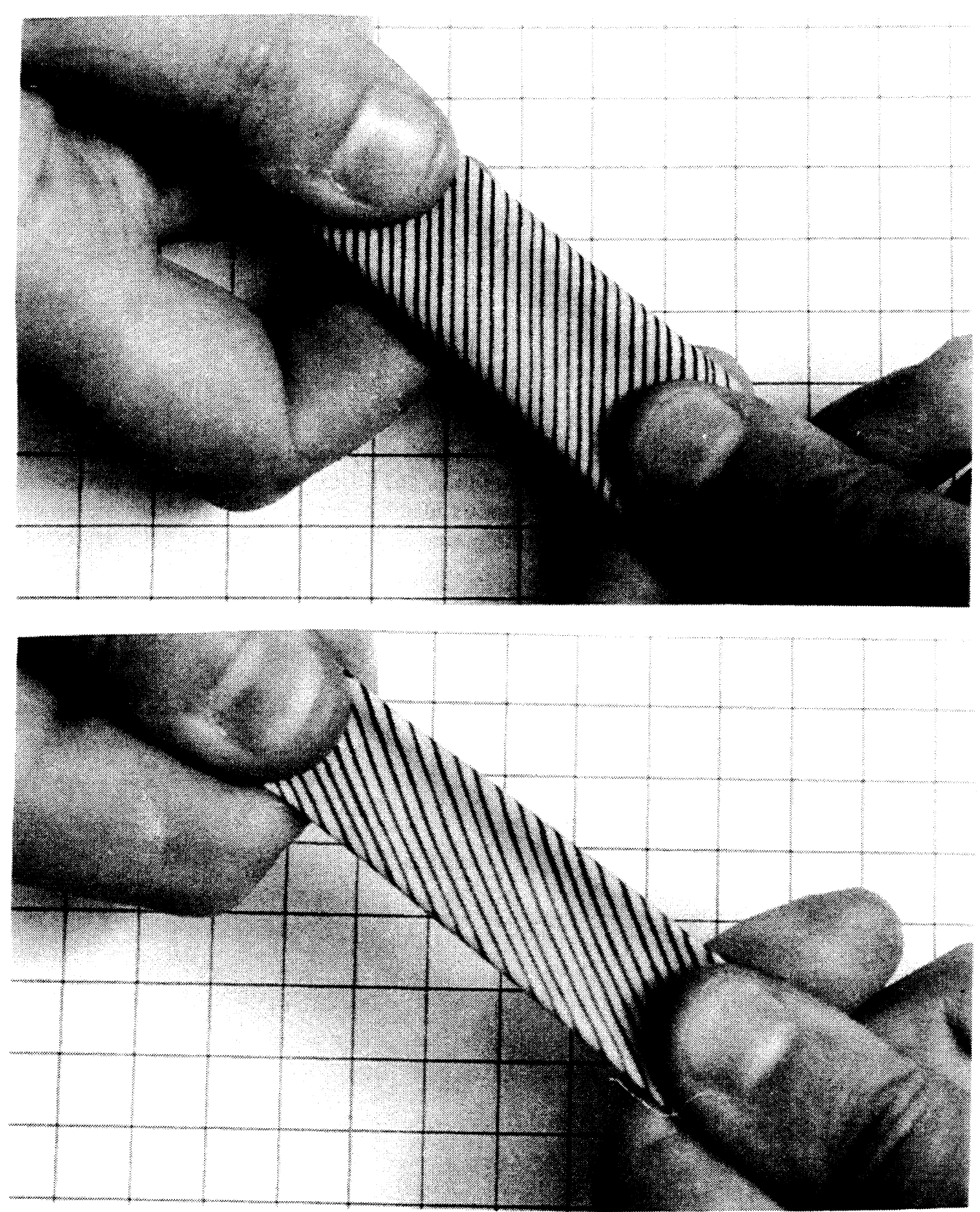

Fig. 5 A rubber band with lines at about $45^{\circ}$ can simulate "capriole". (a) no force applied; (b) tensile force is applied along the longer side: lines will appear rotated showing the "capriola" effect simulating that of Fig.3c. 